\title{
ON EXPLICIT INTERVAL METHODS OF ADAMS-BASHFORTH TYPE
}

\author{
MA MORZATA JANKOWSKA $^{1}$, ANDRZEJ MARCINIAK ${ }^{1,2}$ \\ Poznań University of Technology, Institute of Computing Science \\ Piotrowo 3a, 60-965 Poznań, Poland \\ ${ }^{2}$ Adam Mickiewicz University, Faculty of Mathematics and Computer Science \\ Umultowska 87, 61-614 Poznań, Poland \\ e-mail: anmar@sol.put.poznan.pl
}

\begin{abstract}
In our previous paper [1] we have considered implicit interval multistep methods of AdamsMoulton type for solving the initial value problem. On the basis of these methods and the explicit ones introduced by Sokin [2] we wanted to construct predictor-corrector (explicit-implicit) interval methods. However, it turned out that the formulas given by Šokin are incorrect even in the simplest case. Therefore, in this paper we direct our attention to the explicit interval methods of Adams-Bashforth type and modify the formulas of Šokin. For the modified explicit interval methods it is proved, like for the implicit interval methods considered in [1], that the exact solution of the problem belongs to interval-solutions obtained by these methods. Moreover, it is shown an estimation of the widths of such interval-solutions.
\end{abstract}

\section{INTRODUCTION}

Explicit interval methods for solving the initial value problem have been considered and analyzed in a number of papers and monographs (see e.g. [2] - [11]). Such methods are interesting due to interval-solutions obtained which contain their errors. Computer implementation of the interval methods in floating-point interval arithmetic together with the representation of initial data in the form of machine intervals, i.e. by intervals which ends are equal or neighboring machine numbers, let us achieve interval-solutions which contain all possible numerical errors.

In this paper we direct our attention to the explicit interval methods of Adams-Bashforth type. Such methods have been considered by Šokin [2], Unfortunately, it can be shown that the formulas given by Šokin fail even in the simplest case, i.e. when the number of steps equals 1 (see Sec. 3). For this reason we modified Šokin's formula and showed that methods that we obtained produce the interval-solution that includes the exact solution of the initial value problem.

This paper consists of six sections. In Sec. 2 we define the initial value problem and shortly present the conventional explicit Adams-Bashforth methods. Our modified interval formulas of their conventional equivalents are introduced in Sec. 3. In this section we also show an example of the initial value problem which interval-solution is incorrect when we apply the formulas proposed by Šokin. It is shown that our modified formulas give a correct interval-solution. In Sec. 4 we prove that the exact solution of the initial value problem belongs to interval-solutions obtained by the interval methods of Adams-Bashforth type. In Sec. 5 we estimate the widths of intervalsolutions obtained by the considered interval methods. At the end of our paper we draw some remarks (Sec. 6). 


\section{THE INITIAL VALUE PROBLEM \\ AND CONVENTIONAL ADAMS-BASHFORTH METHODS}

As we know the initial value problem is concerned with finding the solution $y=y(t)$ to a problem of the form

$$
y^{\prime}(t)=f(t, y(t))
$$

subject to an initial condition

$$
y(0)=y_{0}
$$

where $t \in[0, \xi], y \in \mathbf{R}^{N}$ and $f:[0, \xi] \times \mathbf{R}^{N} \rightarrow \mathbf{R}^{N}$. We will assume that the solution of (2.1) exists and is unique.

Let us choose a positive integer $m$ and select the mesh points $i_{0}, i_{1}, \ldots, t_{m}$, where $t_{n}=n h$ for each $n=0,1, \ldots, m$ and $h \quad \xi / m$. Assume that an integer $k=1,2, \ldots$ will state how many approximations $\quad y_{n-k}, \quad y_{n-k+1}, \ldots, \quad y_{n-1} \quad$ of the exact solution at the previous $k$ mesh points have to be known to determine the approximation $y_{n}$ at the point $t_{n}$. form

As shown in [12] the exact solution of (2.1) considered on the interval $\left[t_{n-1}, t_{n}\right]$ has the

$$
y\left(t_{n}\right)=y\left(t_{n-1}\right)+h \sum_{j=0}^{k-1} \gamma_{j} \nabla^{j} f\left(t_{n-1}, y\left(t_{n-1}\right)\right)+h^{k+1} \gamma_{k} \psi(\eta, y(\eta)),
$$

where

$$
\begin{gathered}
\nabla^{j} f\left(t_{n-1}, y\left(t_{n-1}\right)\right)=\sum_{m=0}^{j}(-1)^{m}\left(\begin{array}{c}
j \\
m
\end{array}\right) f\left(t_{n-1-m}, y\left(t_{n-1-m}\right)\right), \\
\gamma_{0}=1, \quad \gamma_{j}=\frac{1}{j !} \int_{0}^{1} s(s+1) \ldots(s+j-1) d s \text { for } j=1,2, \ldots, k,
\end{gathered}
$$

and $\psi(\eta, y(\eta)) \equiv f^{(k)}(\eta, y(\eta)) \equiv y^{(k+1)}(\eta), \quad \eta \in\left[t_{n-k}, t_{n}\right]$.

After replacing the unknown values with $y\left(t_{n-k}\right), \quad y\left(t_{n-k+1}\right), \quad \ldots, \quad y\left(t_{n-1}\right)$ approximations $\quad y_{n-k,} \quad y_{n-k+1}, \quad \ldots, \quad y_{n-1} \quad$ obtained by applying another method (for example by a Runge-Kutta method, see e.g. [12]-[17]) and neglecting the errorterm $h^{k+1} \gamma_{k} \psi(\eta, y(\eta))$ we are given the following formula known as the \&-step implicit Adams-Bashforth method (see also e.g. [12] - [17])

$$
y_{n}=y_{n-1}+h \sum_{j=0}^{k-1} \gamma_{j} \nabla^{j} f_{n-1}
$$

where $f_{n-1}=f\left(t_{n-1}, y_{n-1}\right)$. 


\section{EXPLICIT INTERVAL MULTISTEP METHODS OF ADAMS-BASHFORTH TYPE}

Let us denote:

$$
\begin{gathered}
\Delta_{t}=\{t \in \mathbf{R}: 0 \leq t \leq \xi\}, \\
\Delta_{y}=\left\{y=\left(y_{1}, y_{2}, \ldots, y_{N}\right)^{T} \in \mathbf{R}^{N}: \underline{b}_{i} \leq y_{i} \leq \bar{b}_{i}, \quad i=1,2, \ldots, N\right\},
\end{gathered}
$$

$F(T, Y) \quad$ - an interval extension of $f(t, y) \quad$ (for a definition of interval extension see e.g. [2], [11] or [18]),

$\Psi(T, Y)$ - an interval extension of $\psi(t, y)$.

Let the assumptions about $F(T, Y)$ and $\Psi(T, Y)$ be the same as in [1] and [2], Moreover let us assume that $y(0) \in Y_{0}$ and the intervals $Y_{i}$ such as $y\left(t_{i}\right) \in Y_{i}$ for $i=1,2, \ldots, k-1$ are known. We can obtain such $Y_{i}$ by applying interval one-step method, for example an interval method of Runge-Kutta type (see [19] or [20]). Then the explicit interval method of Adams-Bashforth type constructed by Šokin is given by the formula.

$$
\begin{aligned}
Y_{n}=Y_{n-1} & +h\left(\gamma_{0} F_{n-1}+\gamma_{1} \nabla F_{n-1}+\gamma_{2} \nabla^{2} F_{n-1}+\gamma_{k-1} \nabla^{k-1} F_{n-1}\right) \\
& +h^{k+1} \gamma_{k} \Psi\left(T_{n-1}+[-(k-1) h, 0], Y_{n-1}+[-(k-1) h, 0] F\left(\Delta_{l}, \Delta_{y}\right)\right)
\end{aligned}
$$

for $n=k, k+1, \ldots, m$, where

$$
\begin{gathered}
F_{n-1}=F\left(T_{n-1}, Y_{n-1}\right), \\
\gamma_{0}=1, \quad \gamma_{j}=\frac{1}{j !} \int_{0}^{1} s(s+1) \ldots(s+j-1) d s, \quad j=1,2, \ldots, k, \\
h=\frac{\xi}{\ldots}, \quad t_{i}=i h \in T_{i}, \quad i=0,1, \ldots, m .
\end{gathered}
$$

Unfortunately, it can be shown that the formula (3.1) fails in the simplest case, i.e. when $k=1$. For example, let us consider the initial value problem of the form

where $t \in[0,1]$. The exact solution of this problem is given by

$$
y(t)=\exp (0.5 \mathrm{t})
$$

Applying (3.1) for $m=2000, h=0,0005$ and $Y_{0}=[0,0]$, we get (in floating-point interval arithmetic) the interval-solution of the initial value problem (3.3) at $t=1$ as follows

$$
Y(1)=[1.64872126211491595,1.64872126211491651] .
$$


On the other hand the formula (3.4) yields the exact solution

$$
y_{\text {exact }}(1) \cong 1.64872127070012815 \text {. }
$$

This simple example shows that $y_{\text {exact }}(1) \notin Y(1)$, and hence $Y(1)$ is not the correct intervalsolution of the initial value problem (3.3).

After careful consideration of the Šokin formula (3.1), we have found that the reason for such behavior of the method is defective error term of the form

$$
h^{k+1} \gamma_{k} \Psi\left(T_{n-1}+[-(k-1) h, 0], Y_{n-1}+[-(k-1) h, 0] F\left(\Delta_{t}, \Delta_{y}\right)\right)
$$

As we checked, the formula (3.5) does not improve the interval-solution, and for this reason getting the correct result becomes impossible. Detailed study of conventional Adams-Bashforth methods led us to make some essential modifications. The correct error term should be written as follows

$$
h^{k+1} \gamma_{k} \Psi\left(T_{n-1}+[-(k-1) h, h], Y_{n-1}+[-(k-1) h, h] F\left(\Delta_{t}, \Delta_{y}\right)\right)
$$

Substituting (3.6) into (3.1) we obtain the correct formula for interval methods of Adams-Bashforth type of the following form:

$$
\begin{aligned}
Y_{n}=Y_{n-1} & +h\left(\gamma_{0} F_{n-1}+\gamma_{1} \nabla F_{n-1}+\gamma_{2} \nabla^{2} F_{n-1}+\ldots+\gamma_{k-1} \nabla^{k-1} F_{n-1}\right) \\
& +h^{k+1} \gamma_{k} \Psi\left(T_{n-1}+[-(k-1) h, h], Y_{n-1}+[-(k-1) h, h] F\left(\Delta_{t}, \Delta_{y}\right)\right)
\end{aligned}
$$

where $n=k, k+1, \ldots, m$.

In particular for a given $k$ we get the following methods:

- $k=1 \Rightarrow$

$$
Y_{n}=Y_{n-1}+h F\left(T_{n-1}, Y_{n-1}\right)+\frac{h^{2}}{2} \Psi\left(T_{n-1}+[0, h], Y_{n-1}+[0, h] F\left(\Delta_{l}, \Delta_{y}\right)\right) \text {, }
$$

- $k=2 \Rightarrow$

$$
\begin{aligned}
Y_{n}= & Y_{n-1}+\frac{h}{2}\left(3 F\left(T_{n-1}, Y_{n-1}\right)-F\left(T_{n-2}, Y_{n-2}\right)\right) \\
& +\frac{5 h^{3}}{12} \Psi\left(T_{n-1}+[-h, h], Y_{n-1}+[-h, h] F\left(\Delta_{t}, \Delta_{y}\right)\right),
\end{aligned}
$$

- $k=3 \Rightarrow$

$$
\begin{aligned}
Y_{n}= & Y_{n-1}+\frac{h}{12}\left(23 F\left(T_{n-1}, Y_{n-1}\right)-16 F\left(T_{n-2}, Y_{n-2}\right)+5 F\left(T_{n-3}, Y_{n-3}\right)\right) \\
& +\frac{3 h^{4}}{8} \Psi\left(T_{n-1}+[-2 h, h], Y_{n-1}+[-2 h, h] F\left(\Delta_{t}, \Delta_{y}\right)\right) .
\end{aligned}
$$


Since

$$
\nabla^{j} F\left(T_{n-1}, Y_{n-1}\right)=\sum_{m=0}^{j}(-1)^{m}\left(\begin{array}{c}
j \\
m
\end{array}\right) F\left(T_{n-1-m}, Y_{n-1-m}\right)
$$

the formula (3.7) can be written in the equivalent form

$$
\begin{aligned}
Y_{n}= & Y_{n-1}+h \sum_{j=1}^{k} \beta_{k j} F\left(T_{n-j}, Y_{n-j}\right) \\
& +h^{k+1} \gamma_{k} \Psi\left(T_{n-1}+[-(k-1) h, h], Y_{n-1}+[-(k-1) h, h] F\left(\Delta_{l}, \Delta_{y}\right)\right),
\end{aligned}
$$

where

$$
\beta_{k j}=(-1)^{j-1} \sum_{m=j-1}^{k-1}\left(\begin{array}{c}
m \\
j-1
\end{array}\right) \gamma_{m}, \quad j=1,2, \ldots, k
$$

Now, for comparison, let us apply modified formula (3.7) (or (3.8)) with $k=1$ to the initial value problem (3.3). For $m=2000, h=0,0005$ and $Y_{0}=[0,0]$ we get the interval-solution at $t=1$ as follows

$$
\mathrm{Y}(1)=\left[\begin{array}{lll}
1.6487212621 & 1491595, & 1.64872128787216209
\end{array}\right]
$$

and we have $y_{\text {exact }}(1) \in Y(1)$. Thus, in this case we have obtained the correct interval-solution of the initial value problem (3.3)

\section{THE EXACT SOLUTION VS. INTERVAL SOLUTIONS}

For the method (3.7) we can prove that the exact solution of the initial value problem (2.1) belongs to the intervals obtained by this method. Before that it is convenient to present the following

Lemma 1. If $\left(t_{i}, y\left(t_{i}\right)\right) \in\left(T_{i}, Y_{i}\right)$ for $i=n-k, n-k+1, \ldots, n-1$, where $Y_{i}=Y\left(t_{i}\right)$, than for any $j=0,1, \ldots, k-1$ we have

$$
\nabla^{j} f\left(t_{n-1}, y\left(t_{n-1}\right)\right) \in \nabla^{j} F\left(T_{n-1}, Y_{n-1}\right) .
$$

Proof. Since $F(T, Y)$ is an interval extension of $f(t, y)$, and $\left(t_{i}, y\left(t_{i}\right)\right) \in\left(T_{i}, Y_{i}\right)$ for $i=n-k, n-k+1, \ldots, n-1$, we can write

$$
f\left(t_{n-1-m}, y\left(t_{n-1-m}\right)\right) \in F\left(T_{n-1-m}, Y_{n-1-m}\right), \quad m=0,1, \ldots, j .
$$

This implies that 


$$
\sum_{m=0}^{j}(-1)^{m}\left(\begin{array}{c}
m \\
j
\end{array}\right) f\left(t_{n-1-m}, y\left(t_{n-1-m}\right)\right) \in \sum_{m=0}^{j}(-1)^{m}\left(\begin{array}{c}
m \\
j
\end{array}\right) F\left(T_{n-1-m}, Y_{n-1-m}\right)
$$

But

$$
\sum_{m=0}^{j}(-1)^{m}\left(\begin{array}{c}
j \\
m
\end{array}\right) F\left(T_{n-1-m}, Y_{n-1-m}\right)=\nabla^{j} F\left(T_{n-1}, Y_{n-1}\right)
$$

From (2.3), (4.2) and (4.3) the inclusion (4.1) follows immediately.

Theorem 1. If $y(0) \in Y_{0}$ and $y\left(t_{i}\right) \in Y_{i}$ for $i=1,2, \ldots, k-1$, then for the exact solution $y(t)$ of the initial value problem (2.1) we have

$$
y\left(t_{n}\right) \in Y_{n}
$$

for $n=k, k+1, \ldots, m$, where $Y_{n}=Y\left(t_{n}\right)$ are obtained from the method (3.7).

Proof. Let us consider the formula (2.2) for $n=k$. We get

$$
y\left(t_{k}\right)=y\left(t_{k-1}\right)+h \sum_{j=0}^{k-1} \gamma_{j} \nabla^{j} f\left(t_{k-1}, y\left(t_{k-1}\right)\right)+h^{k+1} \gamma_{k} \psi(\eta, y(\eta))
$$

where $\eta \in\left[t_{0}, t_{k}\right]$. From the assumption we have $y\left(t_{k-1}\right) \in Y_{k-1}$, and from the Lemma 1 it follows that

$$
h \sum_{j=0}^{k-1} \gamma_{j} \nabla^{j} f\left(t_{k-1}, y\left(t_{k-1}\right)\right) \in h \sum_{j=0}^{k-1} \gamma_{j} \nabla^{j} F\left(T_{k-1}, Y_{k-1}\right)
$$

Applying Taylor's formula we have

$$
y(\eta)=y\left(t_{k-1}\right)+\left(\eta-t_{k-1}\right) y^{\prime}\left(t_{k-1}+\vartheta\left(\eta-t_{k}-1\right)\right)
$$

where $\vartheta \in[0,1]$. Because $\eta \in\left[t_{0}, t_{k}\right]$ and $t_{i}=i h$ for $i=0,1, \ldots, m$, we get

$$
\eta-t_{k-1} \in[-(k-1) h, h]
$$

Moreover, $y^{\prime}(t)=f(t, y(t))$. Since

$$
f\left[t_{k-1}+\vartheta\left(\eta-t_{k-1}\right), y\left(t_{k-1}+\vartheta\left(\eta-t_{k-1}\right)\right)\right] \in F\left(\Delta_{t}, \Delta_{y}\right)
$$

then

$$
y^{\prime}\left(t_{k-1}+\vartheta\left(\eta-t_{k-1}\right)\right) \in F\left(\Delta_{t}, \Delta_{y}\right)
$$

Taking into account the above considerations, from the formula (4.5) we get

$$
y(\eta) \in Y_{k-1}+[-(k-1) h, h] F\left(\Delta_{t}, \Delta_{y}\right) .
$$


As we assumed, $\Psi$ is an interval extension of $\psi$. Thus, applying (4.6) and (4.7), we have

$$
\begin{aligned}
& h^{k+1} \gamma_{k} \Psi(\eta, y(\eta)) \\
& \quad \in h^{k+1} \gamma_{k} \Psi\left(T_{k-1}+[-(k-1) h, h], Y_{k-1}+[-(k-1) h, h] F\left(\Delta_{t}, \Delta_{y}\right)\right) .
\end{aligned}
$$

Thus, we have shown that $y\left(t_{k}\right)$ belongs to the interval

$$
\begin{aligned}
Y_{k-1} & +h \sum_{j=0}^{k-1} \gamma_{j} \nabla^{j} F\left(T_{k-1}, Y_{k-1}\right) \\
& +h^{k+1} \gamma_{k} \Psi\left(T_{k-1}+[-(k-1) h, h], Y_{k-1}+[-(k-1) h, h] F\left(\Delta_{1}, \Delta_{y}\right)\right)
\end{aligned}
$$

but - according to the formula (3.7) - this is the interval $Y_{k}$. This conclusion ends the proof for $n=k$. In a similar way we can show the thesis of this theorem for $n=k+1, k+2, \ldots, m$.

\section{WIDTHS OF INTERVAL SOLUTIONS}

Theorem 2. If the intervals $Y_{n}$ for $n=0,1, \ldots, k-1$ are known, $t_{i}=i h \in T_{i}, i=0,1, \ldots, m$, $h=\xi / m$, and $Y_{n}$ for $n=k, k+1, \ldots, m$ are obtained from (3.7), then

$$
d\left(Y_{n}\right) \leq A \max _{q=0,1, \ldots, k-1} d\left(Y_{q}\right)+B \max _{j=1,2, \ldots, m-1} d\left(T_{j}\right)+C h^{k}
$$

where the constants $A, B$ and $\mathrm{C}$ are independent of $h$.

Proof. From (3.8) we get

$$
\begin{aligned}
d\left(Y_{n}\right) \leq & d\left(Y_{n-1}\right)+h \sum_{j=1}^{k}\left|\beta_{k j}\right| d\left(F\left(T_{n-j}, Y_{n-j}\right)\right) \\
& +h^{k+1} \gamma_{k} d\left(\Psi\left(T_{n-1}+[-(k-1) h, h], Y_{n-1}+[-(k-1) h, h] F\left(\Delta_{i}, \Delta_{y}\right)\right)\right)
\end{aligned}
$$

We have assumed that $\Psi$ is monotonic with respect to inclusion. Moreover, if the step size $h$ is such that satisfies the conditions

$$
\begin{aligned}
& T_{n-1}+[-(k-1) h, h] \subset \Delta_{l}, \\
& Y_{n-1}+[-(k-1) h, h] F\left(\Delta_{l}, \Delta_{y}\right) \subset \Delta_{y},
\end{aligned}
$$

then

$$
\Psi\left(T_{n-1}+[-(k-1) h, h], Y_{n-1}+[-(k-1) h, h] F\left(\Delta_{l}, \Delta_{y}\right)\right) \subset \Psi\left(\Delta_{l}, \Delta_{y}\right) .
$$

From (5.3) we have

$$
d\left(\Psi\left(T_{n-1}+[-(k-1) h, h], Y_{n-1}+[-(k-1) h, h] F\left(\Delta_{1}, \Delta_{y}\right)\right)\right) \leq d\left(\Psi\left(\Delta_{1}, \Delta_{y}\right)\right) .
$$


We have also assumed that for the function $F$ there exists a constant $L>0$ such that

$$
d\left(F\left(T_{n-j}, Y_{n-j}\right)\right) \leq L\left(d\left(T_{n-j}\right)+d\left(Y_{n-j}\right)\right)
$$

Therefore, from the inequality (5.2) we get

$$
d\left(Y_{n}\right) \leq d\left(Y_{n-1}\right)+h L \beta_{k} \sum_{j=1}^{k}\left(d\left(T_{n-j}\right)+d\left(Y_{n-j}\right)\right)+h^{k+1} \gamma_{k} d\left(\Psi\left(\Delta_{t}, \Delta_{y}\right)\right),
$$

where

$$
\beta_{k}=\max _{j=1,2, \ldots, k}\left|\beta_{k j}\right|
$$

Denoting

$$
\beta=h L \beta_{k}, \quad \alpha=1+\beta, \quad \gamma=h^{k+1} \gamma_{k},
$$

we can write (5.4) in the form

$$
d\left(Y_{n}\right) \leq \alpha \sum_{j=1}^{k} d\left(Y_{n-j}\right)+\beta \sum_{j=1}^{k} d\left(T_{n-j}\right)+\gamma d\left(\Psi\left(\Delta_{1}, \Delta_{y}\right)\right)
$$

From (5.6) for $\boldsymbol{n}=\boldsymbol{k}$ we have

$$
d\left(Y_{k}\right) \leq \alpha \sum_{j=1}^{k} d\left(Y_{k-j}\right)+\beta \sum_{j=1}^{k} d\left(T_{k-j}\right)+\gamma d\left(\Psi\left(\Delta_{t}, \Delta_{y}\right)\right)
$$

and for $n=k+1$ we get

$$
d\left(Y_{k+1}\right) \leq \alpha d\left(Y_{k}\right)+\alpha \sum_{j=1}^{k-1} d\left(Y_{k-j}\right)+\beta \sum_{j=1}^{k} d\left(T_{k+1-j}\right)+\gamma d\left(\Psi\left(\Delta_{t}, \Delta_{y}\right)\right) .
$$

Applying (5.7) to the above inequality we obtain

$$
\begin{aligned}
d\left(Y_{k+1}\right) \leq & \left(\alpha^{2}+\alpha\right) \sum_{j=1}^{k} d\left(Y_{k}-j\right) \\
& +\beta\left(\alpha \sum_{j=1}^{k} d\left(T_{k-j}\right)+\sum_{j=1}^{k} d\left(T_{k+1-j}\right)\right)+\gamma(\alpha+1) d\left(\Psi\left(\Delta_{l}, \Delta_{y}\right)\right) .
\end{aligned}
$$

From (5.6) for $n=k+2$ we get

$$
d\left(Y_{k+2}\right) \leq \alpha d\left(Y_{k+1}\right)+\alpha d\left(Y_{k}\right)+\alpha \sum_{j=1}^{k-2} d\left(Y_{k-j}\right)+\beta \sum_{j=1}^{k} d\left(T_{k+2-j}\right)+\gamma d\left(\Psi\left(\Delta_{t}, \Delta_{y}\right)\right)
$$


Insertion of (5.7) and (5.8) into this inequality yields

$$
\begin{aligned}
d\left(Y_{k+2}\right) \leq & \left(\alpha^{3}+2 \alpha^{2}+\alpha\right) \sum_{j=1}^{k} d\left(Y_{k}-j\right) \\
& +\beta\left(\left(\alpha^{2}+\alpha\right) \sum_{j=1}^{k} d\left(T_{k}-j\right)+\alpha \sum_{j=1}^{k} d\left(T_{k+1-j}\right)+\sum_{j=1}^{k} d\left(T_{k+2-j}\right)\right) \\
& +\gamma\left(\alpha^{2}+2 \alpha+1\right) d\left(\Psi\left(\Delta_{i}, \Delta_{j}\right)\right) .
\end{aligned}
$$

Now, from (5.6) for $n=k+3$ we get

$$
\begin{aligned}
d\left(Y_{k+3}\right) \leq & \alpha d\left(Y_{k+2}\right)+\alpha d\left(Y_{k+1}\right)+\alpha d\left(Y_{k}\right)+\alpha \sum_{j=1}^{k-3} d\left(Y_{k-j}\right) \\
& +\beta \sum_{j=1}^{k} d\left(T_{k+3-j}\right)+\gamma d\left(\Psi\left(\Delta_{t}, \Delta_{y}\right)\right) .
\end{aligned}
$$

Applying (5.7), (5.8) and (5.9) to the above formula we have

$$
\begin{aligned}
d\left(Y_{k+3}\right) \leq & \left(\alpha^{4}+3 \alpha^{3}+3 \alpha^{2}+\alpha\right) \sum_{j=1}^{k} d\left(Y_{k}-j\right) \\
& +\beta\left(\left(\alpha^{3}+2 \alpha^{2}+\alpha\right) \sum_{j=1}^{k} d\left(T_{k-j}\right)+\left(\alpha^{2}+\alpha\right) \sum_{j=1}^{k} d\left(T_{k+1-j}\right)\right. \\
& \left.+\alpha \sum_{j=1}^{k} d\left(T_{k}+2-j\right)+\sum_{j=1}^{k} d\left(T_{k}+3-j\right)\right) \\
& +\gamma\left(\alpha^{3}+3 \alpha^{2}+3 \alpha+1\right) d\left(\Psi\left(\Delta_{1}, \Delta_{y}\right)\right)
\end{aligned}
$$

Thus, for each $i=1,2, \ldots, m-k$ we have

$$
\begin{aligned}
d\left(Y_{k+i}\right) \leq & \left(\sum_{l=0}^{i}\left(\begin{array}{l}
i \\
l
\end{array}\right) \alpha^{l+1}\right)\left(\sum_{j=1}^{k} d\left(Y_{k}-j\right)\right) \\
& +\beta \sum_{p=0}^{i}\left(\sum_{l=0}^{p-1}\left(\begin{array}{c}
p-1 \\
l
\end{array}\right) \alpha^{l+1}\right)\left(\sum_{j=1}^{k} d\left(T_{k+i-p-j}\right)\right) \\
& +\gamma\left(\sum_{l=0}^{i}\left(\begin{array}{l}
i \\
l
\end{array}\right) \alpha^{l}\right) d\left(\Psi\left(\Delta_{l}, \Delta_{y}\right)\right)
\end{aligned}
$$


Applying the notation (5.5) we obtain

$$
\begin{aligned}
d\left(Y_{k+i}\right) \leq & k \sum_{l=0}^{i}\left(\begin{array}{l}
i \\
l
\end{array}\right)\left(1+h L \beta_{k}\right)^{l+1} \max _{q=0,1, \ldots, k-1} d\left(Y_{q}\right) \\
& +h L \beta_{k} k \sum_{p=0}^{i} \sum_{l=0}^{p-1}\left(\begin{array}{c}
p-1 \\
l
\end{array}\right)\left(1+h L \beta_{k}\right)^{l+1} \max _{j=0,1, \ldots, k+i-1} d\left(T_{j}\right) \\
& +h^{k+1} \gamma_{k} \sum_{l=0}^{i}\left(\begin{array}{l}
i \\
l
\end{array}\right)\left(1+h L \beta_{k}\right)^{l} d\left(\Psi\left(\Delta_{l}, \Delta_{y}\right)\right) .
\end{aligned}
$$

Let us notice that

$$
\begin{gathered}
\left(\begin{array}{l}
i \\
l
\end{array}\right) \leq(m-k) ! \text { for } l=0,1, \ldots, i, \\
\left(\begin{array}{c}
p-1 \\
l
\end{array}\right) \leq(m-k) ! \text { for } l=0,1, \ldots, p-1, \\
\left(1+h L \beta_{k}\right)^{l+1} \leq \exp \left(\xi L \beta_{k}\right), \\
\sum_{l=0}^{p-1}\left(1+h L \beta_{k}\right)^{l+1} \leq \frac{\exp \left(\xi L \beta_{k}\right)-1}{h L \beta_{k}} .
\end{gathered}
$$

On the basis of the above we can make the following estimates

$$
\begin{gathered}
k \sum_{l=0}^{i}\left(\begin{array}{l}
i \\
l
\end{array}\right)\left(1+h L \beta_{k}\right)^{l+1} \leq m(m-k+1) ! \exp \left(\xi L \beta_{k}\right), \\
k \sum_{p=0}^{i} \sum_{l=0}^{p-1}\left(\begin{array}{c}
p-1 \\
l
\end{array}\right)\left(1+h L \beta_{k}\right)^{l+1} \leq m(m-k+1) ! \frac{\exp \left(\xi L \beta_{k}\right)-1}{h L \beta_{k}}, \\
\sum_{l=0}^{i}\left(\begin{array}{l}
i \\
l
\end{array}\right)\left(1+h L \beta_{k}\right)^{l} \leq(m-k+1) ! \frac{\exp \left(\xi L \beta_{k}\right)-1}{h L \beta_{k}} .
\end{gathered}
$$

Thus, from (5.10) we finally get

$$
d\left(Y_{k+i}\right) \leq A \max _{q=0,1, \ldots, k-1} d\left(Y_{q}\right)+B \max _{j=0,1, \ldots, m-1} d\left(T_{j}\right)+C h^{k}
$$

for each $i=0,1, \ldots, m-k$, where

$$
\begin{gathered}
A=m(m-k+1) ! \exp \left(\xi L \beta_{k}\right), \quad B=m(m-k+1) !\left(\exp \left(\xi L \beta_{k}\right)-1\right), \\
C=\frac{\gamma_{k}}{L \beta_{k}}(m-k+1) !\left(\exp \left(\xi L \beta_{k}\right)-1\right) d\left(\Psi\left(\Delta_{l}, \Delta_{y}\right)\right) .
\end{gathered}
$$


Since $T_{0}=[0,0]$, i.e. $\mathrm{d}\left(T_{0}\right)=0$, the inequality (5.1) follows immediately from (5.11).

\section{REMARKS}

The main reason of this paper is directing one attention to the explicit interval multistep methods of Adams-Bashforth type developed earlier by Šokin [2], The implicit multistep methods (of Adams-Moulton type) were presented and carefully analyzed in our previous paper [1]. While building a computational system that would apply both Adams-Bashforth and Adams-Moulton methods some problems with Adams-Bashforth methods appeared. All necessary modifications that had to be made are described in this paper.

At the moment one of our main tasks is to construct multistep predictor-corrector methods and finish an appropriate computer system which would provide interval solutions of all known and constructed multistep methods.

\section{References}

[1] Jankowska, M., Marciniak, A., Implicit Interval Multistep Methodsfor Solving the Initial Value Problem, Computational Methods in Science and Technology 8/1 (2002), 17-30.

[2] Šokin, Ju. I.; Interval Analysis [in Russian], Nauka, Novosibirsk 1981.

[3] Conradt, J., Ein Intervallverfahren zur Einschliessung des Fehlers einer Näherungslösung bei Anfangswertaufgaben für Systeme von gewöhnlichen Differentialgleichungen, Freiburger IntervallBerichte 80/1, Universität Freiburg i. Br. 1980.

[4] Eigenraam, P., The Solution of Initial Value Problems Using Interval Arithmetic, Mathematical Centre Tracts Vol. 144, Mathematisch Centrum, Amsterdam 1981.

[5] Hunger, S., Intervallanalytische Defektabschätzung bei Anfangswertauf gaben für Systeme von gewöhnlichen Differentialgleichungen, Schriften der Gesellschaft für Mathematik und Datenverarbeitung Nr. 41, Bonn 1971.

[6] Kalmykov, S.A., Šokin, Ju. I., Juldašev.Z. H., Solving Ordinaiy Differential Equations by Interval Methods [in Russian], Doklady AN SSSR, Vol. 230, No. 6 (1976).

[7] Kalmykov, S. A., Šokin, Ju. I., Juldašev, Z. H., Methods of Interval Analysis [in Russian], Nauka, Novosibirsk 1986.

[8] Marciniak, A., Finding the Integration Intervalfor Interval Methods ofRunge-Kutta Type in Floating-Point Interval Arithmetic, Pro Dialog 10 (2000), 35^15.

[9] Marcowitz, U., Fehlerabschätzung bei Anfangswertaufgaben für Systeme von gewöhnlichen Differentialgleichungen mit Anwendung aufdas REENTRY-Problem, Numer. Math. 24 (1973).

[10] Marciniak, A.Jnteival Methods ofRunge-KuttaType inFloating-Point Interval Arif/jmerfc [in Polish], Technical Report RB-027/99, Poznan University of Technology, Institute of Computing Science, Poznan 1999.

[11] Moore, R. E., Inteival Analysis, Prentice-Hall, Englewood Cliffs 1966.

[12] Krupowicz, A., Numerical Methods of Initial Value Problems of Ordinaiy Differential Equations [in Polish], PWN, Warsaw 1986.

[13] Butcher, J. C., The Numerical Analysis of Ordinary Differential Equations. Runge-Kutta and General Linear Methods, J. Wiley \& Sons, Chichester 1987.

[14] Dormand, J. R., Numerical Methods for Differential Equations. A Computational Approach, CRC Press, Boca Raton 1996.

[15] Hairer, E., Nørsett, S. P., Wanner, G., Solving Ordinaiy Differential Equations I. Nonstiff Problems, Springer-Verlag, Berlin, Heidelberg 1987.

[16] Matwiejew, N. M., Methods for Integrating Ordinaiy Differential Equations [in Polish], PWN, Warsaw 1982. 
[17] Stetter, H. J., Analysis ofDiscretization Methodsfor Ordinary Differential Equations, Springer-Verlag, Berlin 1973.

[18] Jaulin, L., Kieffer, M., Didrit, O., Walter, É., Applied Interval Analysis, Springer-Verlag, London 2001.

[19] Marciniak, A., Szyszka, B., One- and Two-Stage Implicit Inteiyal MethodsofRunge-Kutta Type, Computational Methods in Science and Technology 5 (1999), 53-65.

[20] Gajda, K., Marciniak, A., Szyszka, B., Three- and Four-Stage Implicit Inteiyal Methods of Runge-Kutta Type, Computational Methods in Science and Technology 6 (2000), 41-59. 